\title{
AUTHOR INDEX (Volume 23)
}

Abadyan, M. 1550101, 1650043

Abbasi, S. A. 1650004

Abd-Alghafour, N. M. 1650057

Abdullah, I. Y. 1650005

Abubakar, D. 1650057

Addie, A. J. 1650044

Adel, C. 1650008

Ahmad, E. 1650026

Ahmad, R. 1650045

Ahmed, N. A. 1650041

Ahmed, N. M. 1650057

Ahmed, S. 1650033

Ahn, B.-H. 1650006

Albesa, A. G. 1650025

Aliofkhazraei, M. 1630001

Allahyarzadeh, M. H. 1630001

An, Y. 1650059

Arseenko, M. Yu. 1550088

Asif M., M. 1650013

Bajat, J. B. 1650030

Bazargan, A. M. 1650014

Bera, A. 1650062

Berrocal, A. 1550091

Bezghiche-Imloul, T. 1650041

Bhatti, M. T. 1550085

Bhowmik, S. 1650033

Bi, J. X. 1550090

Bidadi, H. 1550105

Bououdina, M. 1650057

Brahim, B. 1650008

Brucale, M. 1650058

Bučko, M. 1650030

Cai, C. 1650046

Cao, W. 1650046

Cetinel, A. 1550093

Chadli, R. 1650015

Chai, Y. 1650049

Chakrabarty, D. 1650033

Che, J. 1650010

Chen, C. 1650031

Chen, Q. 1650055

Chen, Y. 1650049
Chen, Y.-Y. 1650039

Chen, Z. 1650034

Cheng, J. 1550080

Chikouche, I. 1550086

Chitra, M. 1550094

Choi, E.-H. 1650039

Chung, G.-S. 1550087

Condurache-Bota, S. 1550104

Constantinescu, C. 1550104

Cui, H. 1650010

Cui, L. 1650010

Cui, W. 1650042

Dai, F. Z. 1650012

Dai, J. 1550102, 1650031

Dai, M. 1650049

Dai, N. 1550110

Dai, X. H. 1650060

Dalouji, V. 1650002

Das, B. 1650056

Das, S. K. 1650040

Deng, D. 1650038

Devarajan, M. 1650016

Djamal, B. 1650050

Dogar, A. H. 1650004

Dong, H.-K. 1550095

Dong, M. 1650047

Doodran, R. J. 1650021

Dou, H. 1550110

Elahi, S. 1650002

Fang, Y. 1550099

Farrokhabadi, A. 1550101

Farshidfar, F. 1650054

Feng, C. 1550111

Feng, K. 1650059

Feng, Y. 1650053

Fethi, B. M. 1650008

Gadhari, P. 1550082

Ganguly, B. 1650056

Gao, J. $\quad$ 1550103, 1550109

Gao, M. 1650034
Gao, Q. 1550099

Gao, X. 1550109

Gao, Y. 1550084

Ghorbanian, B. 1650021

Ghosh, I. 1650056

Girija, E. K. 1550094

Gu, L. 1550098

Guan, Y. 1630003

Guo, J. 1550100

Guo, J. D. 1550090

Guo, J. X. 1650060

Hajivaliei, M. 1650052

Hammache-Makhloufi, H. 1650041

Han, P. 1630002

Han, S. 1550111

Hao, X. 1550098

Hassan, A. I. 1650044

Hassan, Z. 1550106, 1650057

He, L. 1550103

He, Y. $\quad$ 1550092, 1550108

He, Z. 1550109, 1650010, 1650011

Hei, H. $\quad$ 1550109, 1650011

Honey, S. 1550085

Hosseinzadeh, S. 1550105

Hou, J. 1550100

Hu, J. 1650049

Huang, B. 1650055

Huang, H. 1650034

Huang, J. 1650034

Huang, X. 1650032

Huang, Y. 1550098

Huang, Z. 1650046

Huo, D. 1550110

Hussain, F. 1650026

Hussain, M. S. 1650004

Huttula, M. 1650046

Ikonen, K. 1650042

Imran, M. 1650026

Iqbal, F. 1650026

Ishaq, A. 1550085

Ismail, N. I. 1650035 
Jeong, Y. S. 1650006

Ji, H. 1550103

Ji, N. 1550081

Jia, Y. L. 1650060

Jiang, D. 1650048, 1650061

Jiang, J. 1550111

Jiang, Y. 1550097

Ju, X. 1650048

Jumali, M. H. H. 1650005

Kaciulis, S. 1650058

Kakhaki, Z. M. 1650014

Kanani, A. 1650043

Kariper, İ. A. 1650009

Karthikeyan, N. 1550089

Kazemzad, M. 1650054

Kazemzadeh, A. 1650014

Keivani, M. 1550101, 1650043

Kermanpur, A. 1550083

Khalid, S. 1650024

Khalifa, Z. S. 1650001

Khanlarkhani, A. 1650054

Khashan, K. S. 1650044

Khater, A. 1650015

Kheffache, S. 1650015

Khoie, S. Md. M. 1650021

Khoso, N. A. 1650003

Kirveslahti, A. $\quad 1650022,1650042$

Kishi, N. 1650027

Kolisnichenko, O. V. 1550088

Kong, H. 1650047

Koo, B.-H. 1650006

Koochi, A. 1550101, 1650043

Korhonen, T. 1650022

Kovaleva, M. G. 1550088

Kumari, N. 1650036

Kundu, M. 1550092

Lai, M. 1650032

Lefkaier, I. K. 1650050

Le Gall, R. 1650050

Legat, A. 1650030

Lei, X. L. 1550108

Leng, X. $\quad 1650020,1650061$

Li, C. 1550103

Li, D. 1650048

Li, J. $\quad$ 1550100, 1550107

Li, M. 1650034

Li, R. 1550092

Li, S. 1550102

Li, W. 1650020, 1650059

Li, X. H. 1650060

Lim, W. Q. 1650016

Lin, N. $\quad 1630002,1550092$

Lin, Z. 1550096

Ling, J. 1650061

Liu, B. T. 1650060
Liu, F. 1550099

Liu, G. $\quad$ 1550107, 1650028

Liu, H. $\quad$ 1550081, 1650038

Liu, J. 1650011

Liu, K. 1550081

Liu, L. $\quad$ 1650023, 1650047

Liu, Q. 1650034

Liu, S. 1550103

Liu, W. 1550099

Liu, X. 1550109, 1650017, 1650020, 1650047, 1650061, 1650099

Lou, J. Z. 1650060

Lu, F. 1650023

Lu, J. Z. 1650012

Lu, Y. 1650020, 1650061

Ma, G. 1550092

Ma, Y. 1630002

Ma, Z. 1550092

Maaza, M. 1550085

Makhloufi, L. 1650030

Maldonado, F. 1650037

Mamunin, K. N. 1550088

Memon, H. $\quad 1650003,1650036$

Memon, S. 1650003

Mezzi, A. 1650058

Miao, Y. 1650059

Mielonen, K. 1650042

Min, F. 1650023

Mishra, B. K. 1650056

Mo, R. 1550103

Moya, R. 1550091

Mukherjee, S. 1650033

Muñoz, F. 1550091

Murtaza, G. 1650045

Naderi, N. 1650014

Naseem, S. 1550085

Nasresfahani, M. R. 1550083

Nath, R. K. 1650056

Naz, M. Y. 1650035

Nazari, S. 1650052

Neelakandeswari, N. 1550094

Nie, F. 1550103

Niroumand, B. 1550083

Niu, T. 1650038

Ouyang, R. 1650059

Özcelik, Z. 1550093

Paczkowska, M. 1650007

Padiyan, D. P. 1550094

Pakkanen, T. A. 1550097, 1650022, 1650042

Pal, A. 1650056

Panja, B. 1650040

Pavlenko, I. A. 1550088
Perumal, R. 1550106

Piasecki, A. 1650007

Praisler, M. 1550104

Prozorova, M. S. 1550088

Qayyum, A. 1650004

Qi, T. 1650011

Qian, H. 1650032

Qian, W. 1550111

Qian, Z.-J. 1550103

Qiao, Y. $\quad$ 1650011, 1650018

Qiu, J. 1650028

Qu, K. L. 1650019

Raeissi, M. 1550083

Rafti, M. 1650025

Rahmati, A. 1550105

Rajasekaran, N. 1550094

Rashid, M. 1650026

Rashid, N. M. 1650027

Rasouli, M. 1650021

Rehman, Z. U. 1650006

Ren, P. 1550107

Ren, Y. 1550098

Rezaei, M. 1650054

Rodriguez-Solis, M. 1550091

Rouhaghdam, A. S. 1630001

Saadat, S. 1650045

Saha, B. 1650062

Sahari, A. 1550086

Sahoo, P. $\quad 1650040,1550082$

Sangpour, P. 1650014

Sathiya, P. 1650013

Sattar, A. 1650026

Sedighi, H. M. 1550101

Selech, J. 1650007

Sereika, R. 1650058

Shafique, M. A. 1650045

Shah, Z. H. 1650024

Shahedin, A. M. 1550101

Shahnawaz, M. 1650045

Shanmugan, S. 1650016

Shanshool, H. M. 1650005

Shao, H. 1550103

Shao, Z. 1650029

Sharif, R. 1650024

Shen, B. 1550096

Shen, X. 1650029

Shen, Y. 1650011

Shi, J. 1650060

Shi, L. B. 1550095

Shi, X. 1650051

Shrikrishna, K. A. 1650013

Shukrullah, S. 1650035

Sirota, V. V. 1550088

Soga, T. 1650027 
Song, J.-I. 1650006

Song, S. 1650023

Song, X. 1650047

Starbird, R. 1550091

Stashans, A. 1650037

$\mathrm{Su}, \mathrm{C} . \quad 1550100$

$\mathrm{Su}, \mathrm{W} . \quad 1550110,1550103$

Sulaiman, S. A. 1650035

Sun, B. 1650018

Sun, F. 1550096

Sun, F. H. 1550108

Sun, Q. 1650038

Sun, R. 1630003

Sun, Z. 1650034

Suvanto, M. 1550097, 1650022, 1650042

Tang, B. 1550092, 1550109, 1630002

Tang, Y. 1650018

Thema, F. T. 1550085

Tian, W. 1630002

Tigau, N. 1550104

Touazi, S. 1650030

Tyurin, N. Yu. 1550088

Uddin, A. S. M. I. 1550087

Uddin, M. K. H. 1650045

Uhm, H.-S. 1650039

Ullah, H. 1650026

Uthayarani, K. 1550094

Vasilik, N. Ya. 1550088

Vicente, J. L. 1650025

Vignesh, K. 1550089

Vijayalakshmi, K. A. 1550089

Wang, G. 1650046

Wang, J. 1650060
Wang, L. 1650020, 1650047, 1650061

Wang, M. 1550092

Wang, Q.-X. 1650055

Wang, S. 1650018

Wang, W. 1550107, 1650032

Wang, X. 1550092

Wang, X. H. 1650019

Wang, Y. 1650010, 1650018

Wang, Y.-P. 1550095

Wang, Z. 1630002, 1630002, 1650017,1650020

Wang, Z. K. 1650019

Wen, X.-F. 1550080

Wen, Y. 1650032

Weng, F. 1650031

Wolfman, J. 1650028

$\mathrm{Wu}, \mathrm{C} . \quad 1650010$

Wu, H. T. 1550090

Xie, A.-G. 1650039

Xie, R. 1630002

Xiong, J. 1550080

$\mathrm{Xu}$, C. 1650032

Xu, Y. 1550081

Xue, Z. 1550098

Yahaya, M. 1650005

Yang, G. 1650010

Yang, H. 1550080

Yang, Y. 1650059

Yapryntsev, M. N. 1550088

Yasin, S. 1650003

Ye, F. 1550111

Ye, M. 1550100

Yong, H. 1650032

You, K. 1650017

Youzbashi, A. 1650014

Yu, G. $\quad 1550110$

Yu, H. 1650031
Yu, L. 1550111

Yu, S. $\quad$ 1550109, 1650011, 1650017

Zaheer, Z. 1650045

Zegadi, A. 1550086

Zhang, C. 1650053

Zhang, D. 1550107

Zhang, E.-G. 1650055

Zhang, F. 1650029

Zhang, H. 1650046

Zhang, L. 1650038

Zhang, M. 1650023

Zhang, P. 1550103

Zhang, S. 1550099

Zhang, W. 1650059

Zhang, X. Y. 1650060

Zhang, Y. 1650017,1550080

Zhang, Y. K. 1650012

Zhang, Z. D. 1650012

Zhao, F. 1650034

Zhao, Q. X. 1650060

Zhao, R. 1650029

Zheng, K. 1550109

Zheng, R. 1550098

Zheng, S. 1550084

Zhon, L. 1550100

Zhong, Q. 1550111

Zhong, Q. D. 1650051

Zhou, J. Z. 1650012

Zhou, N. 1550103

Zhou, P. 1630002

Zhou, S. $\quad 1650048,1650059$

Zhou, W. 1550099, 1650048

Zhou, Y. 1650032

Zhu, J. 1550102, 1650031

Zhu, Y. 1630003

Zhuang, L. 1550102

Zong, T. 1650059

Zou, J. 1630002

Zouaoui, A. 1550086 